\section{Miguel J.-M. Lewin}

\section{Remerciements}

L'auteur exprime ses remerciements au Pr Mi chel Mignon et au Dr Annick Thomas-Soumarmon pour leurs commentaires, à M. Gabriel Peranzi pour la réalisation des préprarations et photographies de microscopie électronique et à Mme Fabienne Bonfils pour son aide précieuse dans la mise en forme du manuscrit.

\section{ADRESSE}

M. I.-M. Lewin : direclenur de recherche ì l'lnserm, directeur de l'unilé 10) de 'Inserm de gastroentérologie. Inserm U. 10, Hôpital Bichat, 170, boulevard Ney, 75877 Paris (Cedex 18,

\title{
Les inhibiteurs de la pompe à protons gastrique : mode d'action et intérêt thérapeutique
}

Au cours de ces vingt dernières années, le traitement des maladies liées à la sécrétion acide de l'estomac, comme l'ulcère gastro-duodénal et l'œsophagite par reflux, a été considérablement amélioré par le développement de médicaments inhibant la stimulation physiologique de cette sécrétion (antagonistes du récepteur de l'histamine de type H2). Les inhibiteurs de la pompe à protons (IPP) constituent une nouvelle classe de molécules dont l'action antisécrétoire, remarquablement efficace et prolongée, repose sur un mécanisme différent : le blocage du transport gastrique du proton $\mathrm{H}^{+}$par l'ATPase $\left(\mathrm{H}^{+}, \mathbf{K}^{+}\right)$.

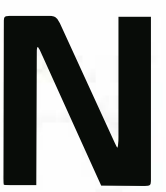
otre estomac possède la remarquable propriété de sécréter de l'acide chlorhydrique en solution très concentrée ( $\mathrm{pH}$ $<2)$. Cette sécrétion acide provient des cellules " pariétales " situées dans les replis glandulaires de l'épithélium fundique (partie haute de l'estomac) [1]. Elle est sous le contrôle d'une régulation nerveuse centrale utilisant le nerf vague comme voie de transmission et d'une régulation locale faisant intervenir de nombreux médiateurs dont, particulièrement, l'histamine et la gastrine [2]. L'une de ses principales fonctions est d'entamer la digestion protéolytique des aliments en transformant le pepsinogène gastrique en pepsine et en maintenant un $\mathrm{pH}$ propice à l'activité de cette enzyme. Normalement, l'estomac ne se digère pas lui-même car les cellules qui tapissent sa surface sécrètent un film protecteur de mucus et produisent aussi des ions bicarbonates qui neutralisent localement les ions $\mathrm{H}^{+}$. Cette barrière de défense n'est cependant pas infaillible. Lorsqu'elle vient à céder, la muqueuse est soumise à une attaque protéolytique qui peut conduire à la formation d'ulcères au niveau de l'estomac ou du duodénum. Une inflammation plus ou moins sévère de l'épithélium œsophagien (œsophagite) peut également survenir lorsqu'un mauvais fonctionnement du sphincter inférieur de l'œsophage permet au contenu gastrique de refluer vers le haut $[3,4]$. Ulcères et œesophagites sont responsables de douleurs et de handicaps socio-professionnels pouvant altérer sévèrement la qualité de vie et sont surtout susceptibles d'évoluer vers de graves complications. Un ulcère non soigné peut aboutir à une perforation de la paroi digestive ou à une hémorragie. A plus long terme, le contact chronique avec le suc gas- 


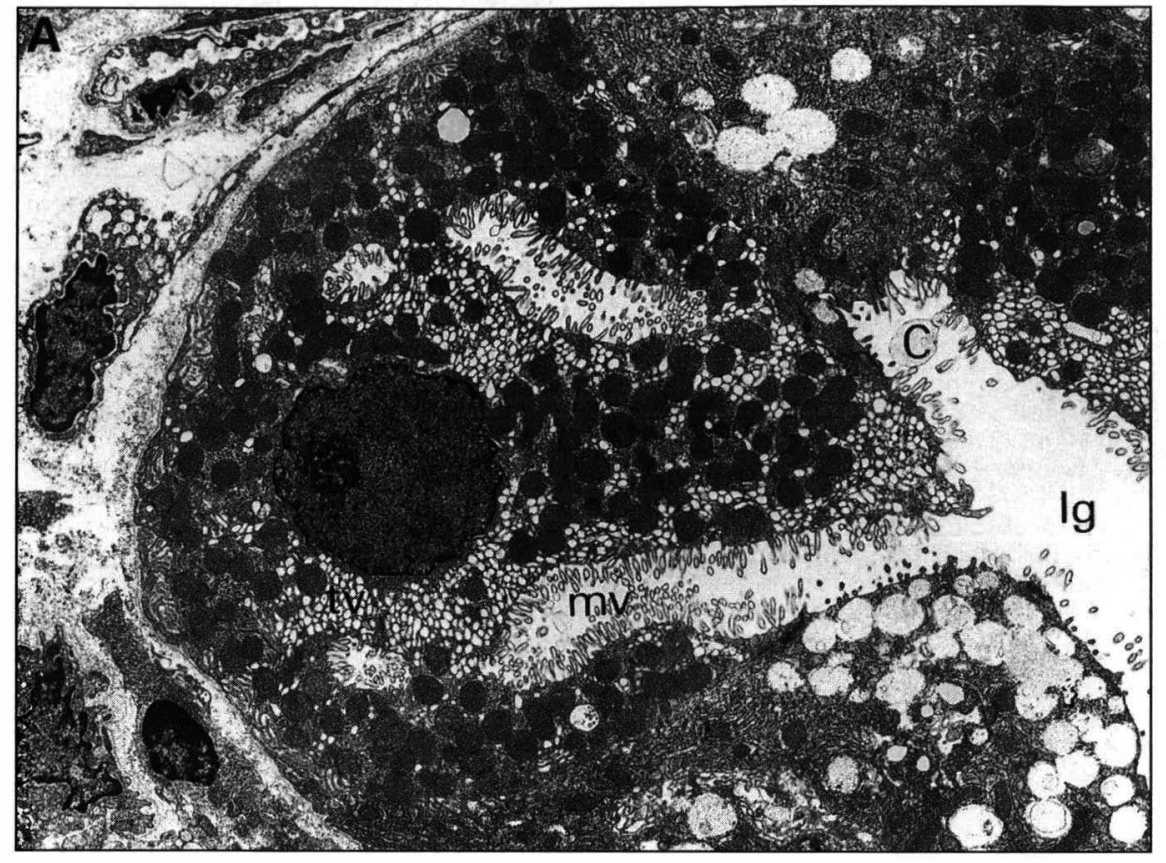

Figure 1. A: Cellule pariétale gastrique vue au microscope électronique. Cette cellule qui est stimulée par l'histamine montre peu de tubulovésicules intracytoplasmiques (tv) et un canalicule sécrétoire très développé (c) bordé de nombreuses microvillosités (mv) s'ouvrant sur la lumière gastrique (lg). $B$ : Cryofracture d'une portion du canalicule sécrétoire montrant les particules d'ATPase (flèches) dans la membrane des microvillosités (mv).

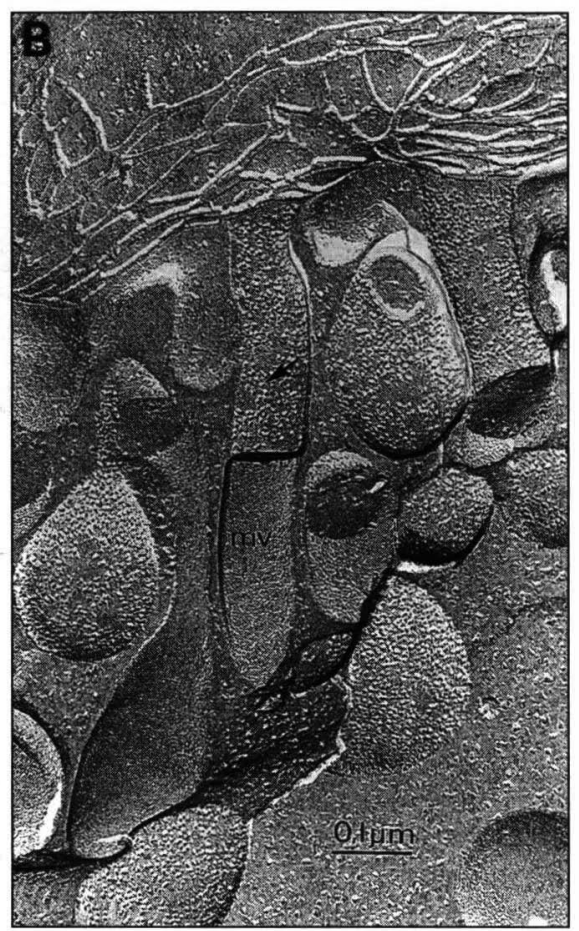

gastro-duodénaux cicatrisent spontanément s'ils sont mis pendant quelques semaines à l'abri de la sécrétion acide gastrique. Dans le cas des œesophagites par reflux, on constate aussi une réparation spontanée de l'épithélium en absence de contact avec l'acide gastrique. Pendant longtemps, la neutralisation des ions $\mathrm{H}^{+}$par des substances "antiacides ", a été, faute de mieux, le seul traitement médical connu. L'inhibition de la stimulation de la cellule pariétale, beaucoup plus efficace, a été rendue possible en 1972 avec la caractérisation pharmacologique du récepteur histaminique $\mathrm{H} 2$ et du premier médicament antagoniste de ce récepteur, la cimétidine, par l'équipe du prix Nobel Jim Black [7]. Nous ne nous étendrons pas davantage sur les médicaments de cette famille qui ont déjà fait l'objet d'une mise au point dans cette revue [8]. Parallèlement à la caractérisation pharmacologique, 


\section{RÉFÉRENCES}

1. Helander HF. The cells of the gastric mucosa. Int Rev Cytol 1981 ; 70 : $217-90$.

2. Lewin MJM. Parietal cell receptors of acid secretion. In: Domschke W, Konturek SJ, eds. Stomach and its diseases. Berlin : Springer, $1993: 3-21$.

\section{Lewin MJM. Estomac. Encyclopedia Univer-} salis $1993: 829-32$.

4. Galmiche JP, Bruley des Varannes S. Reflux gastro-œesophagien et œesophagite : physiopathologie, symptomes, diagnostic et traitement. In: Mignon M. ed. Gastroentérologie. Paris : Ellipses/Aupelf, 1992 : 223-41.

5. Poynard T. Incidence, prévalence, morbidité, mortalité de la maladie ulcéreuse duodénale en France. In : Prise en charge au long cours du patient ulcéreux duodénal. Rev Prat $1992 ; 6$ (suppl.) : 7-10.

6. Sonnenberg A, Sonnenberg GS. Epidemiology of ulcer disease. In: Domschke W, miology of ulcer disease. In: Domschke W, Berlin : Springer, 1993 : 215-27.

7. Black JW, Duncan WA, Durant GJ, Gannellin CR, Parsons ME. Definition and antagonism of histamine H2-receptors. Nalure $1972 ; 236: 385-90$

8. Bonfils S. Les antisécrétoires gastrigues : présent et futur. médecine/sciences $1985 ; 1$ 241-7.

9. Lewin MJM. La sécrétion acide de l'estomac : un mécanisme biologique peu commun. médecine/sciences $1985 ; 1$ : 23440.

10. Ganser AL, Forte JG. K-stimulated ATPase in purified microsomes of bullfrog ATPase in purified microsomes of bullfrog
oxyntic cells. Biochim Biophys Acta 1973 ; 307: $169-80$.

11. Sachs G, Chang HH, Rabon E, Schackman R, Lewin MJM, Saccomani G. A nonelectrogenic $\mathrm{H}^{+}$pump in plasma membranes of hog stomach. I Biol Chem 1976 ; $251: 7690-8$

12. Lewin MJM. Molecular mechanisms of gastric $\mathrm{HCl}$ secretion. In : Desnuelle P, ed. Molecular and cellular biology of digesiton. Amsterdam : Elsevier, $1986: 507-56$

13. Rabon EC, Reuben MA. The mechanism and structure of the gastric H,K-ATPase. Annu Rev Physiol 1990 ; 52 : 321-44.

14. Klaasen CHW, De Pont JJHHM. Gastric $\mathrm{H}^{+} / \mathrm{K}^{+}$-ATPase. Cell Physiol Biochem 1994; 4 : 115-34.

15. Nelson N. Structure and pharmacology of the proton-ATPases. Trends Pharmacol So
16. Crowson MS, Shull GE. Isolation and characterization of a cDNA encoding the putative distal colon H,K-ATPase. Similarity of deduced amino acid sequence to gastric $\mathrm{H}, \mathrm{K}-\mathrm{ATP}$ ase and Na,K-ATPase and mRNA expression in distal colon, kidney, and uterus. J Biol Chem 1992 ; 267 : 13740-8.

17. Jaisser F, Horisberger JD, Geering $\mathrm{K}$, Rossier $\mathrm{BC}$. Mechanisms of urinary $\mathrm{K}^{+}$and $\mathrm{H}^{+}$excretion : primary structure and functional expression of a novel $\mathrm{H}, \mathrm{K}$-ATPase. $J$ Cell Biol 1993; 123 : $1421-9$

18. Jaisser F, Coutry N, Farman N, Binder $\mathrm{HJ}$, Rossier BC. A putative H,K-ATPase is selectively expressed in surface epithelial cells of rat distal colon. Am J Physiol 1993 ; 265 : C1080-9.

19. Peranzi G, Bayle D, Lewin MJM, Soumarmon $\mathrm{A}$. The intramembranous particles of resting and secreting gastric $\left(\mathbf{H}^{+}, \mathbf{K}^{+}\right)$ ATPase membranes. Biol Cell $1991 ; 73$ : 163 71.

20. Lewin MJM. Cell physiology and pharmacology of gastric acid secretion. Thérapie $1992 \cdot 47 \cdot 93-6$

21. Rabon E, Gunther RD, Soumarmon A Bassilian S, Lewin MIM, Sachs G. Solubilization and reconstitution of the gastric H,K ATPase. J Biol Chem 1985; 260 : 10200-7.

22. Soumarmon A, Grelac F, Lewin MIM Solubilization of active $\left(\mathrm{H}^{+}, \mathrm{K}^{+}\right)$-ATPase from gastric membrane. Biochim Biophys Acta $1983 ; 732: 579-85$.

23. Rabon EC, Gunther RD, Bassilian $S$ Kempner ES. Radiation inactivation analysis of oligomeric structure of the $\mathrm{H}, \mathrm{K}$ ATPase.J Biol Chem 1988 ; 263 : 16189-94.

24. Shull GE, Lingrel JB. Molecular cloning of the rat stomach $\left(\mathrm{H}^{+}, \mathrm{K}^{+}\right)$ATPase. J Bio Chem $1986 ; 261$ : 16788-91.

25. Shull GE. cDNA cloning of the $\beta$-subu nit of the rat gastric $\mathrm{H}, \mathrm{K}$-ATPase.J Biol Chem $1990 ; 265: 12123-6$.

26. Reuben MA, Lasater LS, Sachs G. Characterization of a $\beta$ subunit of the gastric $\mathrm{H}^{+} / \mathrm{K}^{+}$-transporting ATPase. Proc Natl Acad Sci USA $1990 ; 87: 6767-71$.

27. Canfield VA, Okamoto CT, Chow D Dorfman J, Gros P, Forte JG, Levenson R. Cloning of the H,K-ATPase $\beta$-subunit : tissue-specific expression, chromosomal assignement, and relationship to Na,K-ATPase 3 subunits. J Biol Chem $1990 ; 265$ : 19878-84.

28. Song I, Yamada T, Trent JM. Mapping of the gene encoding the $\alpha$-subunit of the human $\mathrm{H}^{+}, \mathbf{K}^{+}$-ATPase to chromosome

$19 \mathrm{q} 13.1$ by fluorescent in situ hybridization. Genomics $1992 ; 14: 547-8$. puis moléculaire, des récepteurs histaminiques $\mathrm{H} 2$ et des autres récepteurs de la cellule pariétale, des progrès considérables ont été réalisés dans la connaissance du mécanisme sécrétoire lui-même. Nous avons déjà décrit l'essentiel de ce mécanisme dans un précédent numéro [9]. Dans la suite de cet article, nous nous proposons de faire le point des connaissances actuelles sur l'ATPase $\left(\mathrm{H}^{+}, \mathrm{K}^{+}\right)$, responsable du transport gastrique de $\mathrm{H}^{+}$et sur les nouveaux médicaments antisécrétoires inhibiteurs de cette enzyme.

\section{L'ATPase $\left(H^{+}, K^{+}\right)$, pompe à protons de la cellule pariétale}

La concentration de l'ion $\mathrm{H}^{+}$dans le suc gastrique est un million de fois plus élevée que dans le sang. La nature du système de transport capable d'établir un tel gradient n'a été révélée qu'en 1973 avec la découverte d'une ATPase stimulée par l'ion $\mathrm{K}^{+}$ dans les microsomes de muqueuse gastrique [10]. Une série de travaux, principalement conduits par l'équipe de G. Sachs aux Etats-Unis, allait montrer que cette ATPase était caractéristique de la cellule pariétale et pouvait transporter $\mathrm{H}^{+}$en utilisant $\mathrm{K}^{+}$ comme contre-ion, d'où son appellation de «pompe à protons" ou d'"ATPase $\left(\mathrm{H}^{+}, \mathrm{K}^{+}\right) »[11-14]$.

L'ATPase $\left(\mathrm{H}^{+}, \mathrm{K}^{+}\right)$gastrique fait partie des ATPases dont le fonctionnement repose sur un mécanisme de phosphorylation et dénommées pour cette raison «P-ATPases". Les P-ATPases transportant $\mathrm{H}^{+}$sont apparues très tôt dans l'évolution puisqu'on les trouve dans diverses bactéries et levures (Neurospora crassa, Saccharomyces cerevisiae, Schizosaccharomyces pombe) et même dans le règne végétal [15]. Les ATPases $\left(\mathrm{Na}^{+}, \mathrm{K}^{+}\right)$et $\mathrm{Ca}^{2+}$ font partie de cette même famille. Cependant, l'ATPase $\left(\mathrm{H}^{+}, \mathrm{K}^{+}\right)$gastrique se distingue par plusieurs caractéristiques. En particulier, contrairement à l'ATPase $\left(\mathrm{Na}^{+}, \mathrm{K}^{+}\right)$, elle n'est pas inhibée par l'ouabaïne et son fonctionnement est électriquement neutre. Par ailleurs, elle diffère de l'ATPase $\mathrm{H}^{+}$mitochondriale et des ATPases $\mathrm{H}^{+}$"vacuolaires " ( V-ATPases») présentes sur les vésicules d'endocytose, les granules sécrétoires et les lysosomes puisque le fonction- 


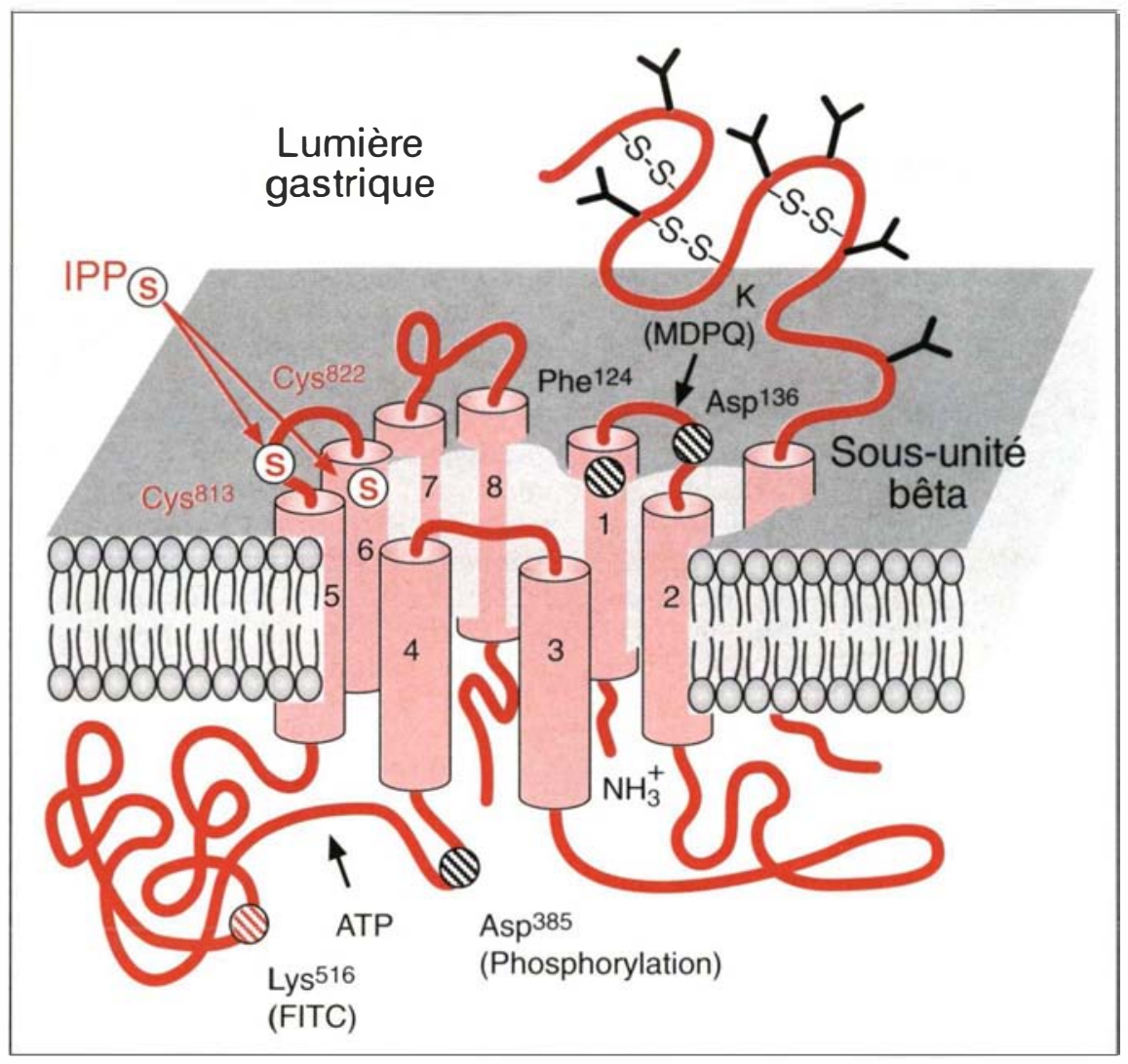

Figure 2. Représentation hypothétique de l'ATPase $\left(H^{+}, K^{+}\right)$gastrique dans la membrane du canalicule sécrétoire de la cellule pariétale. La sous-unité $\alpha$ (représentée ici avec 8 domaines transmembranaires) pourrait former une poche hydrophile évoquant un canal par lequel $\mathrm{H}^{+}$pourrait être transporté. Un autre canal se formerait entre la sous-unité $\alpha$ et la sous-unité $\beta$ pour le transport de $K^{+}$. La région d'interaction avec l'ATP, sur la partie cytosolique de la sous-unité $\alpha$ située entre le quatrième et le cinquième domaine transmembranaire, comprend l'acide aspartique en position 385 (Asp385) qui est le site de phosphorylation de l'enzyme et la lysine en position 516 (Lys516) où se fixe la sonde fluorescente FITC. La région fixant l'ion $\mathrm{K}^{+}$, de l'autre côté de la membrane, comprend la phénylalanine en position 124 (Phe124) située dans le premier domaine transmembranaire et l'acide aspartique en position 136 (Asp136) situé dans la première boucle extracellulaire. Cette région fixe aussi la sonde fluorescente MDPQ. Elle est en interaction allostérique avec la région fixant I'ATP. Les IPP sous leur forme activée (sulfénamides) établissent des ponts S-S avec les cystéines en position 813 (Cys813) et 822 (Cys822). Une fixation sur un autre résidu cystéine (Cys892) a été également mise en évidence pour l'oméprazole et le lansoprazole mais pas pour le pantoprazole. Elle ne semble, par conséquent, pas spécifique de l'effet inhibiteur et n'a donc pas été représentée. Remarquons que les IPP ne se fixent pas sur les cystéines de la partie extracellulaire de la sous-unité $\beta$ qui ne sont pas libres. nement de ces ATPases est électrogénique et n'implique pas de réaction covalente de phosphorylation. Contrairement à ce que l'on a initialement cru, l'ATPase gastrique n'est pas unique en son genre car des ATPases de type $\left(\mathrm{H}^{+}, \mathrm{K}^{+}\right)$ont été récemment caractérisées au niveau du côlon et du rein [16-18]. Cependant les structures moléculaires de ces ATPases sont sensiblement différentes de celle de l'enzyme gastrique $(65 \%$ d'analogie de séquences d'acides aminés). La distribution tissulaire de l'ATPase $\left(\mathrm{H}^{+}, \mathrm{K}^{+}\right)$gastrique a été examinée à l'aide d'anticorps monoclonaux et par hybridation avec des sondes nucléotidiques. Ces deux approches indiquent une localisation exclusive au niveau de la cellule pariétale. En particulier, l'ATPase $\left(\mathrm{H}^{+}, \mathrm{K}^{+}\right)$gastrique n'est pas détectée au niveau du rein ou du côlon et, réciproquement, les ATPases $\left(\mathrm{H}^{+}, \mathrm{K}^{+}\right)$ identifiées dans ces organes ne sont pas détectées au niveau de l'estomac. 1)ans la cellule pariétale au repos, l'ATPase $\left(\mathrm{H}^{+}, \mathrm{K}^{+}\right)$est mise en réserve sur des «tubulovésicules" intracytoplasmiques. Lorsque la sécrétion acide est stimuléc, ces tubulovésicules migrent vers le pôle apical et fusionnent pour former un "canalicule sécrétoire " bordé de microvillosités où se trouve alors concentrée toute l'ATPase, représentant plus de $85 \%$ des protéines membranaires $[1,2,9$, 19, 20] (figurp 1, A el B).

Comme les ATPases $\left(\mathrm{Na}^{+}, \mathrm{K}^{+}\right)$, les ATPases $\left(\mathrm{H}^{+}, \mathrm{K}^{+}\right)$comprennent deux sous-unités distinctes, $\alpha$ et $\beta$, associées sous forme dimérique $\alpha \beta$ ou tétramérique, $(\alpha \beta)_{2,}$ [21-27].

Le gène humain de la sous-unité $\alpha$ de l'ATPase $\left(\mathrm{H}^{+}, \mathrm{K}^{+}\right)$gastrique a été localisé sur le bras long du chromosome 19 , à côté de celui codant pour l'isoforme $\alpha 3$ de l'ATPase $\left(\mathrm{Na}^{+}, \mathrm{K}^{+}\right)$ de type neuronal [28]. Ces deux sous-unités présentent $64 \%$ de similitude de séquence. On pense qu'elles proviennent de la divergence d'un gène ancestral commun, il $y$ a quelques centaines de millions d'années. Le gène humain de la sous-unité $\beta$ a été localisé sur le bras long du chromosome 13 [29]. Sa structure est très proche de celle des gènes codant pour les isoformes $\beta 1$ et $\beta 2$ de l'ATPase $\left(\mathrm{Na}^{+}, \mathrm{K}^{+}\right)$. Ces gènes sont cependant situés sur un chromosome différent (chromosome 1), avec ceux 


\section{RÉFÉRENCES}

29. Song I, Brown DR, Yamada T, Trent JM. Mapping of the gene encoding the $\beta$-subunit of $\mathrm{H}^{+}, \mathrm{K}^{+}$-ATPase to human chromosome $13 \mathrm{q} 34$ by fluorescence in situ hybridization. Genomics $1992 ; 14: 1114-5$.

30. Maeda M, Oshiman KI, Tamura S, Futa M. Human gastric $\left(\mathrm{H}^{+} \mathrm{K}^{+}\right)$-ATPase gene. Similarity to $\left(\mathrm{Na}^{+} \mathrm{K}^{+}\right)$-ATPase genes in control region. 9 Biol Chem $1990 ; 265: 9027$ 32.

31. Maeda M, Oshiman KI, Tamura S,Kaya $S$, Mahmood S, Reuben MA, Lasater LS Sachs G, Futai $\mathrm{M}$. The rat $\mathrm{H}^{+} / \mathrm{K}^{+}$-ATPase $\beta$ subunit gene and recognition of its control region by gastric DNA binding protein. I Biol Chem 1991 ; 266 : 215848

32. Newman PR, Shull GE. Rat gastric $H, K$ ATPase $\beta$-subunit gene : intron/exon organization, identification of multiple transcription initiation sites, and analysis of the 5'-flanking region. Genomics 1991, 11: 252 62

33. Sachs G, Munson K, Balaji VN, Aures-Fischer D, Hersey SJ, Hall K. Functional domains of the gastric HK ATPase. J Bioenerg Biomembr 1989 ; 21 : 573-88.

34. Forte JG, Chow DC. Structural and func tional significance of the gastric $\mathrm{H}^{+}, \mathrm{K}^{+}$ ATPase. In: Domschke W, Konturek SI, eds. Stomach and its diseases. Berlin : Springer, $1993: 22-37$

35. Horisberger JD, Jaunin P, Reuben MA Lasater LS, Chow DC, Forte JG, Sachs G Rossier BC, Geering K. The H,K-ATPase $\beta$ subunit can act as a surrogate for the $\beta$ subunit of the Na,K pumps. I Biol Chem $1991 ; 266$ : 19131-4

36. Eakle KA, Seong K, Kabalin MA, Far ley RA. High-affinity ouabain binding by yeast cells expressing $\mathrm{Na}^{+}, \mathrm{K}^{+}$-ATPase $\alpha$ subunits and the gastric $\mathrm{H}^{+}, \mathrm{K}^{+}$-ATPase $\beta$ subunit Proc Natl Acad Sci USA 1992 ; 89 : 2834-8.

37. Chow DC, Browning CM, Forte JG. Gastric $\mathrm{H}^{+}, \mathrm{K}^{+}$-ATPase activity is inhibited by re duction of disulfide bonds in $\beta$-subunit. $A m$ J Physiol 1992 ; 263 : C39-46.

38. Chow DC, Forte JG. Characterization of the $\beta$-subunit of the $\mathrm{H}^{+}-\mathrm{K}^{+}$-ATPase using an inhibitory monoclonal antibody. Am JPhysiol $1993 ; 265 \cdot$ C1562-70.

39. Shin JM, Sachs G. Identification of a region of the $H, K-A T P a s e ~ \alpha$ subunit associated with the $\beta$ subunit. I Biol Chem 1994 ; 269 : 8642-6.

40. Wallmark B, Stewart HB, Rabon E, Sac comani G, Sachs G. The catalytic cycle of gastric $\left(\mathrm{H}^{+}-\mathrm{K}^{+}\right)$-ATPase. I Biol Chem 1980; codant pour les isoformes $\alpha$ l de l'ATPase $\left(\mathrm{Na}^{+}, \mathrm{K}^{+}\right)$rénale, insensible à l'ouabaïne, et l'isoforme $\alpha 2$ présente dans de nombreux organes, dont l'estomac.

Les gènes des sous-unités $\alpha$ et $\beta$ de l'ATPase $\left(\mathrm{H}^{+}, \mathrm{K}^{+}\right)$contiennent dans leur région régulatrice des motifs qui pourraient expliquer leur co-expression spécifique dans la cellule pariétale. La région régulatrice du gène de la sous-unité $\alpha$ contient aussi des sites récepteurs pour l'hormone thyroïdienne et les glucocorticoïdes ainsi que des motifs CRE (cyclic AMP-responsive elements) [30-32].

Le squelette protéique de la sous-unité $\beta$ est codé par un $\mathrm{ADNc}$ de $1,4 \mathrm{~kb}$ et représente un enchaînement de 291 acides aminés d'une masse moléculaire de $34 \mathrm{kDa}$. Il est ancré à la membrane plasmique par un seul domaine transmembranaire précédé d'une courte queue intracellulaire amino-terminale de 40 acides aminés. La partie carboxy-terminale, extracellulaire, est très riche en résidus $\mathrm{N}$-glycosylés, ce qui explique la masse moléculaire apparente plus élevée (60 à $80 \mathrm{kDa})$ observée en électrophorèse. Cette partie contient aussi six cystéines associées deux par deux par des ponts disulfure. On retrouve ces cystéines, aux mêmes positions, dans toutes les sous-unités $\beta$ d'ATPases [26, 27] (figure 2). La fonction de ces sous-unités n'est pas encore bien comprise [33, 34]. On sait qu'elles sont nécessaires à l'expression in vitro des sous-unités $\alpha$. Pour cette expression, les sous-unités $\beta$ des ATPases $\left(\mathrm{H}^{+}, \mathrm{K}^{+}\right)$et $\left(\mathrm{Na}^{+}, \mathrm{K}^{+}\right)$sont, dans une certaine mesure, interchangeables $[35,36]$. Par analogie avec l'ATPase $\left(\mathrm{Na}^{+}, \mathrm{K}^{+}\right)$, on pense que la sous-unité $\beta$ de l'ATPase $\left(\mathrm{H}^{+}, \mathrm{K}^{+}\right)$ contrôle le routage de la sous-unité $\alpha$ du réticulum endoplasmique vers le réseau golgien puis la membrane apicale. Il se pourrait aussi que la sousunité $\beta$ assure le bon repliement de la sous-unité $\alpha$ et stabilise sa structure dans une conformation fonctionnelle. Dans cette stabilisation, l'une au moins des six cystéines amino-terminales joue un rôle crucial puisque les agents réducteurs des liaisons S-S inhibent l'activité ATPasique [37]. Cette activité peut être aussi inhibée par des anticorps monoclonaux dirigés contre des épitopes cytoplasmiques de $\beta$, ce qui suggère une implication plus étendue de cette molécule [34, 38]. Une étude récente, combinant la digestion enzymatique et la solubilisation par des détergents, a montré que la sous-unité $\beta$ copurifiait avec des fragments protéolysés de sousunité $\alpha$ dont la nature variait selon que l'ion $\mathrm{K}^{+}$était présent ou non. Il existerait donc une association fonctionnelle entre les deux sous-unités $\alpha$ et $\beta$, en relation avec le transport de $\mathrm{K}^{+}[37,39]$. Enfin, grâce à ses résidus glycosylés, la sous-unité $\beta$ pourrait jouer un rôle protecteur pour la sous-unité $\alpha$ contre l'activité protéolytique de la pepsine [34].

La sous-unité $\alpha$ est la structure "active " de l'ATPase $\left(\mathrm{H}^{+}, \mathrm{K}^{+}\right)$remplissant à la fois la fonction enzymatique et celle de transport d'ions. C'est une longue protéine de 1034 acides aminés $(110 \mathrm{kDa})$ qui présente au moins 8 domaines transmembranaires (/igure 2). Son fonctionnement peut être schématiquement décrit comme une alternance entre deux états conformationnels : un état E1, non phosphorylé, possédant des sites cytosoliques de faible affinité pour $\mathrm{K}^{+}$, et un état E2, phosphorylé, présentant des sites extracellulaires de forte affinité pour $\mathrm{K}^{+}$. Chaque cycle catalytique transporte 1 (ou 2) $\mathrm{H}^{+}$vers la lumière gastrique et 1 (ou 2) $\mathrm{K}^{+}$dans l'autre sens. L'échange étant stœchiométrique, le transport net est électriquement neutre. Comme $\mathrm{K}^{+}$ est nécessaire au transport de $\mathrm{H}^{+}$, on pense que l'ATPase $\left(\mathrm{H}^{+}, \mathrm{K}^{+}\right)$est associée, sur la membrane sécrétoire, à un canal permettant le transport de $\mathrm{K}^{+}$dans la lumière gastrique, ce transport étant lui-même électriquement couplé à un transport de $\mathrm{Cl}^{-}$à travers un autre canal. Au total, puisque l'ion $\mathrm{K}^{+}$est recyclé, le système produit de l'acide chlorhydrique (figure 3) $[12,13,38]$.

Dans ce mécanisme, deux domaines moléculaires jouent un rôle particulièrement important : le domaine fixant $\mathrm{l}^{\prime}$ ion $\mathrm{K}^{+}$, localisé au niveau de la première boucle extracellulaire et le domaine fixant l'ATP localisé dans la deuxième boucle intracellulaire (figure 2). Le marquage de ces domaines par deux sondes fluorescentes, respectivement le MDPQ (1-(2 méthylphényl)-4 méthylamino6-méthyl-2,3-dihydropyrrolo [3,2-c] quinoléine) et le FITC (isothiocyanate de fluorescéine), suggère qu'ils se 


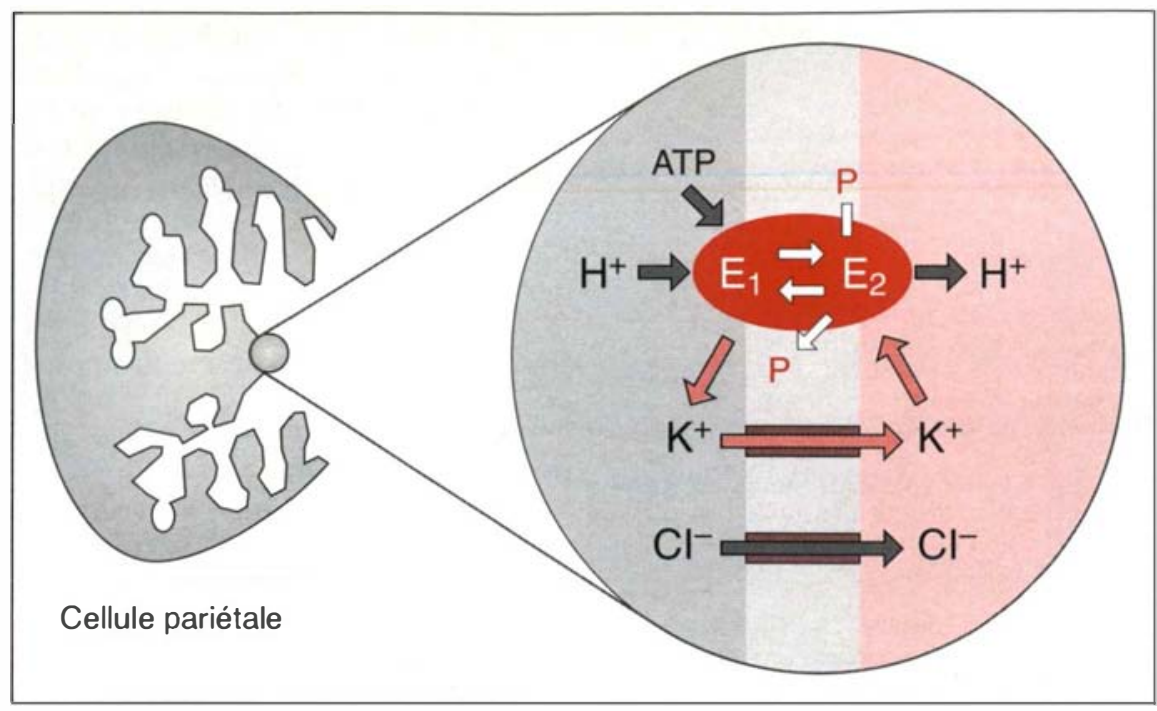

déplacent au cours du cycle catalytique : la fixation de $\mathrm{H}^{+}$, du côté cytosolique, rapprocherait le site ATP de la membrane sécrétoire provoquant une augmentation de la fluorescence du FITC (par augmentation d'hydrophobicité de l'environnement) tandis que la phosphorylation qui suit l'hydrolyse de l'ATP enfoncerait le site luminal de $\mathrm{K}^{+}$dans cette membrane, provoquant ainsi une augmentation de la fluorescence du MDPQ. La fixation de $\mathrm{K}^{+}$sur la face luminale de l'enzyme et la déphosphorylation qui s'ensuit produiraient des effets opposés [13, 33, 40, 41]. Ce modèle, qui reste encore bien sûr hypothétique, est cohérent avec une observation déjà ancienne mettant en évidence une augmentation de fluorescence d'une sonde protéique anionique, l'ANS (aminonaphtol sulfonate de sodium), lors du transport de $\mathrm{H}^{+}$par les microsomes gastriques [42]. Il est également en accord avec les études de protéolyse ménagée et celles analysant la fixation d'anticorps spécifiques. Ces deux approches suggèrent en effet qu'il existe des différences de répartition intra/extramembranaire et de structures tertiaires entre les formes $\mathrm{El}$ et E2 [43, 44]. Le transport ionique par l'ATPase $\left(\mathrm{H}^{+}, \mathrm{K}^{+}\right)$impliquerait donc une dynamique conformationnelle amenant les sites $\mathrm{H}^{+}$et $\mathrm{K}^{+}$à se rapprocher et à s'éloigner l'un de l'autre, alternativement. On verra que les IPP font précisément obstacle à cette dynamique.

Les mécanismes biochimiques qui $m / s n^{\circ}$ I, vol. II, janvier 95 sous-tendent le processus de migration et de fusion des tubulovésicules ne sont pas encore totalement élucidés. Ils impliquent vraisemblablement des réactions de phosphorylation contrôlées par les concentrations intracellulaires du $\mathrm{Ca}^{2+}$ et de l'AMPc. Une fois l'ATPase mise en place sur la membrane sécrétoire, son fonctionnement est déclenché par le transport de $\mathrm{Cl}^{-}$, entraînant celui de $\mathrm{K}^{+}[45,46]$. On sait que l'ouverture du canal transportant $\mathrm{Cl}^{-}$est contrôlée par l'AMPc [47]. Une certaine concentration intracellulaire de ce nucléotide paraît donc nécessaire à l'activation physiologique de l'ATPase $\left(\mathrm{H}^{+}, \mathrm{K}^{+}\right)$. La présence de CRE sur le gène de la sous-unité $\alpha$ indique que l'AMPc interviendrait aussi dans la régulation transcriptionnelle de son expression. Les sous-unités $\alpha$ et $\beta$ contiennent des sites consensus de phosphorylation par les protéines kinases dépendantes du $\mathrm{Ca}^{2+}$ mais l'existence d'une régulation physiologique s'exerçant directement sur l'activité ATPasique n'a pas été démontrée. Un contrôle métabolique de cette activité, par exemple via la phosphorylation oxydative (produisant l'ATP), ne semble pas, non plus, être opérationnel. Il apparaît donc que c'est l'ATPase $\left(\mathrm{H}^{+}, \mathrm{K}^{+}\right)$elle-même qui doit être la cible pharmacologique des médicaments visant à inhiber spécifiquement le transport de $\mathrm{H}^{+}$gastrique.
Figure 3. Mécanisme du transport de $\mathrm{HCl}$ par la membrane du canalicule sécrétoire. Ce schéma montre l'alternance des états E1 et E2 de l'ATPase $\left(\mathrm{H}^{+}, \mathrm{K}^{+}\right)$assurant le contre-transport $\mathrm{H}^{+} / \mathrm{K}^{+}$et le recyclage de $\mathrm{K}^{+}$à travers un canal couplé à un canal transportant $\mathrm{Cr}$.

\section{Les inhibiteurs de l'ATPase $\left(\mathrm{H}^{+}, \mathrm{K}^{+}\right)$ gastrique}

Deux types d'inhibiteurs de l'ATPase $\left(\mathrm{H}^{+}, \mathrm{K}^{+}\right)$gastrique ont été à ce jour caractérisés. Le premier correspond à des molécules entrant en compétition avec l'ion $\mathrm{K}^{+}$pour se fixer sur la face luminale de l'enzyme. Le prototype en est le composé "SCH 28080" à structure imidazo-pyridinique. Les molécules de ce type pourraient permettre d'envisager la mise au point de médicaments inhibiteurs de l'ATPase $\left(\mathrm{H}^{+}, \mathrm{K}^{+}\right)$gastrique à action réversible. Cependant, leur développement pharmaceutique est encore à un stade très préliminaire [48-50].

Le deuxième type d'inhibiteurs correspond à une série de composés dont la structure est dérivée de celle du benzimidazole. Ils constituent la classe nouvelle IPP. Le chef de file en est l'oméprazole $\left(\right.$ Mopral $^{\circledR}$ ou Zoltum $^{\circledR}$ en France, Losec ${ }^{\circledR}$ aux ÉtatsUnis) suivi par le lansoprazole (Lanzor $^{\circledast}$ ou Ogast ${ }^{\circledR}$ ), le pantoprazole et d'autres "...zole" encore en cours de mise au point.

Le mécanisme d'action de tous ces IPP est essentiellement le même : une inhibition totale et irréversible de l'activité de transport de l'ATPase $\left(\mathrm{H}^{+}, \mathrm{K}^{+}\right)$due à une liaison covalente avec la sous-unité $\alpha$, qui "paralyse» l'enzyme en configuration E2 [51, 52].

A l'état de base, ces médicaments sont des promédicaments inactifs. Le groupe sulfoxyde qui est à l'origine 


\section{RÉFÉRENCES}

41. Sachs G, Besancon M, Shin JM Mercier F, Munson K, Hersey S. Structural aspects of the gastric H,K-ATPase. I Bioenerg Biomemlr 1992; 24 : 301-8.

42. Lewin M, Saccomani G, Schackmann R Sachs G. Use of 1-anilino-8-naphthalene-sulfonate as a probe of gastric vesicle transport. I Memlr Biol 1977; 32 : 301-18.

43. Bayle D, Robert JC, Bamberg K, Benkouka F, Cheret AM, Lewin MJM, Sachs G, Soumarmon A. Location of the cytoplasmic epitope for a $\mathrm{K}^{+}$-competitive antibody of the $\left(\mathrm{H}^{+}, \mathrm{K}^{+}\right)$-ATPase. J Biol Chem 1992; 267 $19060-5$.

44. Mercier F, Bayle D, Besancon M, Joys T, Shin JM, Lewin MJM, Prinz C, Reuben MA Soumarmon A, Wong $\mathrm{H}$, Walsh JH, Sachs G. Antibody epitope mapping the gastric $\mathrm{H}^{+} / \mathrm{K}^{+}$-ATPase. Biochim Biophys Acta 1993 $1149: 151-65$

45. Wolosin JM, Forte JG. Changes in the membrane environment of the $\left(\mathrm{K}^{+} \mathrm{H}\right)$ ATPase following stimulation of the gastric oxyntic cell. I Biol Chem 1981 ; 256 : 3149-52.

46. Wolosin JM, Forte JG. Stimulation of oxyntic cell triggers $\mathrm{K}^{+}$and $\mathrm{Cl}^{-}$conductances in $(\mathrm{H}, \mathrm{K})$-ATPase membrane. $A m j$ Physiol 1984 ; 246 : C.537-45.

47. Soumarmon A, Abastado $M$, Bonfils S, Lewin MJM. CI transport in gastric microsomes. J Biol Chem 1980; $255: 11682-7$.

48. Wallmark B, Briving C, Fryklund I, Munson K, Fackson R, Mendlein J, Rabon E, Sachs G. Inhibition of gastric $\mathrm{H}^{+}, \mathrm{K}^{+}$-ATPase and acid secretion by $\mathrm{SCH} 28080$, a substituted pyridyl(1,2a)imidazole. I Biol Chem $1987 ; 262: 2077-84$.

49. Ife RJ, Brown TH, Keeling DJ, Leach CA, Meeson ML, Parsons ME, Reavill DR, Theobald CJ, Wiggall KJ. Reversible inhibitors of the gastric $\left(\mathrm{H}^{+}, \mathrm{K}^{+}\right)$-ATPase. 3. 3-substituted4-(phenylamino)quinolines. I Med Chem 1992 ; 35 : 3413-22.

50. Freston JW. Future prospects for proton pump inhibitors. Aliment Pharmacol Ther 1993; 7 (suppl 1) : 68-75.

51. Fellenius E, Berglindh T, Sachs C, Olbe L, Elander B, Sjostrand SE, Wallmark B. Substituted benzimidazoles inhibit gastric acid secretion by blocking $\left(\mathrm{H}^{+}, \mathrm{K}^{+}\right)$ATPase. Nalure $1981 ; 290: 159-61$.

52. Wallmark B. Mechanism of action of omeprazole. Scand J Gastroenterol 1986;21 (suppl 118) : 11-7.

53. Im WB, Sih JC, Blakeman DP, McGrath JP. Omeprazole, a specific inhibitor of gastric $\left(\mathrm{H}^{+}, \mathrm{K}^{+}\right)$-ATPase, is a $\mathrm{H}^{+}$-activated oxidizing agent of sulfhydryl groups. I Biol Chem
54. Besancon M, Shin JM, Mercier F, Munson $K$, Miller M, Hersey S, Sachs G. Membrane topology and omeprazole labeling of the gastric $\mathrm{H}^{+}, \mathrm{K}^{+}$-adenosinetriphosphatase. Biochemistry $1993 ; 32: 2345-55$.

55. Qhin JM, Besancon M, Simon A, Sachs $G$. The site of action of pantoprazole in the gastric $\mathrm{H}^{+} / \mathrm{K}^{+}$-ATPase. Biochim Biophys Acta 1993 ; 1148 : 223-33.

56. Sachs G, Munson K, Hall K, Hersey SJ. Gastric $\mathbf{H}^{+}, \mathbf{K}^{+}$-ATPase as a therapeutic target in peptic ulcer disease. Dig Dis Sci $1990 ; 35$ : 1537-44.

57. Sachs G, Shin JM, Besancon M, Prinz C. The continuing development of gastric acid pump inhibitors. Aliment Pharmacol Ther pump inhibitors. A 7 (suppl 1) : 4-12.

58. Lindberg $\mathbf{P}$, Brandstrom A, Wallmark B. Structure-activity relationships of omeprazole analogues and their mechanism of action. Trends Pharmacol Si 1987 ; 8 : 399-402.

59. Regardh CG, Andersson T, Lagerstrom PO, Lundborg P, Skanberg I. The pharmacokinetics of omeprazole in humans. A study of single intravenous and oral doses. Ther Drug Monit 1990 ; 12 : 163-72.

60. Barradell LB, Faulds D, McTavish D. Lansoprazole. A review of its pharmacodynamic and pharmacokinetic properties and its therapeutic efficacy in acid-related disorders. Drugs $1992 ; 44: 225-50$.

61. Borg KO, Olbe L. Omeprazole. A survey of preclinical data. Scand I Gastroenterol $1985 ; 20$ (suppl) : 108 .

62. Shamburek RD, Schubert ML. Control of gastric acid secretion - histamine H2-receptor antagonists and $\mathbf{H}^{+} \mathbf{K}^{+}$-ATPase. Gastroenterol Clin North Am 1992; 21 : 527-50.

63. Lewin MJM. Oméprazole : un nouveau mécanisme d'action et une efficacité sans précédent dans le traitement de l'hypersécrétion acide gastrique. Gastroenterol clin Biol $1984 ; 8$ : 939-42.

64. Nagaya H, Inatomi N, Satoh H. Differences in the antisecretory actions of the proton pump inhibitor AG-1749 (Lansoprazole) and the histamine H2-receptor antagonist famotidine in rats and dogs. Jap J Pharmacol 1991 ; 55 : 425-36.

65. Maton PN. Omeprazole. N Engl J Med $1991 ; 324: 965-75$

66. Cadranel JF, Ruszniewski Ph, ElouaerBlanc L, Lehy T, Delchier JC, Cadiot G, Florent Ch, Vatier J, Mignon M. Efficacité et tolérance à long terme de l'oméprazole chez 20 malades présentant un syndrome de Zollinger-Ellison sévère. Gastroenterol Clin Biol $1989 ; 13: 654-62$. de leur pouvoir inhibiteur doit d'abord être réduit pour engendrer un cycle sulfénamide. Ce cycle sulfénamide possède un soufre réactif qui peut alors établir des liaisons S-S avec des cystéines libres de la sous-unité $\alpha$ (figure 2). La cible pharmacologique des IPP est donc située sur la face luminale du canalicule sécrétoire et la transformation de ces médicaments en forme sulfénamide active a lieu dans ce même canalicule, sous l'influence des ions $\mathrm{H}^{+}$.

Leur structure chimique confère aux IPP des propriétés de bases faibles. De plus, grâce à l'addition de divers radicaux sur les cycles benzymidazoliques et pyridiniques, leur pK (constante d'ionisation) a été ajusté à des valeurs inférieures à 4. Les IPP sont donc pratiquement non dissociés à $\mathrm{pH}$ neutre, très peu dissociés dans les compartiments cellulaires modérément acides (comme les lysosomes, les vésicules d'endocytose et les granules sécrétoires) et totalement dissociés (forme cationique) dans le canalicule sécrétoire. Cette caractéristique leur pormet de pénétrer librement dans toutes les cellules, à l'état de promédicament électriquement neutre, et de s'accumuler spécifiquemcnt, sous forme protonéc, dans le canalicule de la cellule pariétale puis de se transformer en forme active au voisinage même de l'ATPase. Ainsi, le mécanisme d'action de ces inhibiteurs comporte trois étapes s'enchaînant rapidement : accumulation, activation et fixation covalente [51-58] (figures 4 et 5).

Le rôle clé joué par la protonation dans le mécanisme d'action des IPP explique la très grande spécificité de ces médicaments. En effet, le canalicule de la cellule pariétale est le seul compartiment biologique dont le $\mathrm{pH}$ soit suffisamment bas pour que l'accumulation et l'activation des IPP puissent se produire. En outre, l'ATPase $\left(\mathrm{H}^{+}, \mathrm{K}^{+}\right)$est la protéine très largement majoritaire du canalicule sécrétoire. De plus, la durée de vie de la forme sulfénamide active libre est très brève et la réaction de liaison avec les groupes SH est limitée à l'environnement immédiat : ainsi, l'oméprazole et le lansoprazole n'ont aucun effet in vivo sur les ATPases $\left(\mathrm{H}^{+}, \mathrm{K}^{+}\right)$du côlon et du rein bien que ces ATPases présentent une certaine 


\begin{tabular}{lllll}
\hline Omeprazole & $\mathrm{R}_{1}$ & $\mathrm{OCH}_{3}$ & $\mathrm{CH}_{3}$ & $\mathrm{OCH}_{3}$ \\
\hline Lansoprazole & $\mathrm{OCH}_{2}$ & $\mathrm{CF}_{3}$ & $\mathrm{CH}_{3}$ \\
\hline Pantoprazole & $\mathrm{OCH}_{3}$ & $\mathrm{OCH}_{3}$ & $\mathrm{OCHF}_{2}$ \\
\hline
\end{tabular}

Figure 4. Structure chimique des IPP actuellement développés.

sensibilité à ces antagonistes in vitro. Enfin, les IPP sont rapidement métabolisés par le foie avec une demi-vie sanguine de l'ordre de deux heures Ils sont donc rapidement épurés des divers compartiments biologiques sauf du canalicule acide des cellules pariétales où se situe précisément leur site d'action.

Ces caractéristiques pharmacodynamiques concourent aussi à minimiser le risque d'éventuels effets secondaires. Ceux-ci se limitent à une interaction, d'importance relativement modeste, avec la biosynthèse de certaines isoformes du cytochrome P45(), vraisemblablement à cause de la présence du cycle imidazolique dans la molécule (en pratique, il faut éviter d'associer les IPP avec certains médicaments comme ceux à base de phénytoïne, de théophylline ou d'antivitamine $\mathrm{K}$, dont l'élimination pourrait être ralentie ) [59-62].

In rivo, les ponts S-S liant la forme activée des inhibiteurs benzimidazoliques à l'ATPase sont irréversibles. Comme le temps de demi-renouvellement de cette enzyme est de l'ordre de 36 heures, la durée d'action des IPP est extrêmement longue et se prolonge bien au-delà de leur présence dans le sang. Cette propriété pharmacocinétique particulière distingue la classe des IPP de celle des inhibiteurs des récepteurs $\mathrm{H} 2$ dont les effets, réversibles, sont proportionnels à la concentration sanguine $[63,64]$.

Puisque la pompe à protons est le dernier maillon du mécanisme de la sécrétion acide, les IPP sont capables d'inhiber cette sécrétion quels que soient le niveau et la nature de sa stimulation. lls sont donc totalement et universellement efficaces, contrairement aux antagonistes du récepteur H2 dont l'action se limite à l'inhibition de la voie de stimulation histaminique. Puisque cette voie de stimulation joue un rôle prépondérant (c'est elle qui est responsable de la production d'AMPc dans la cellule

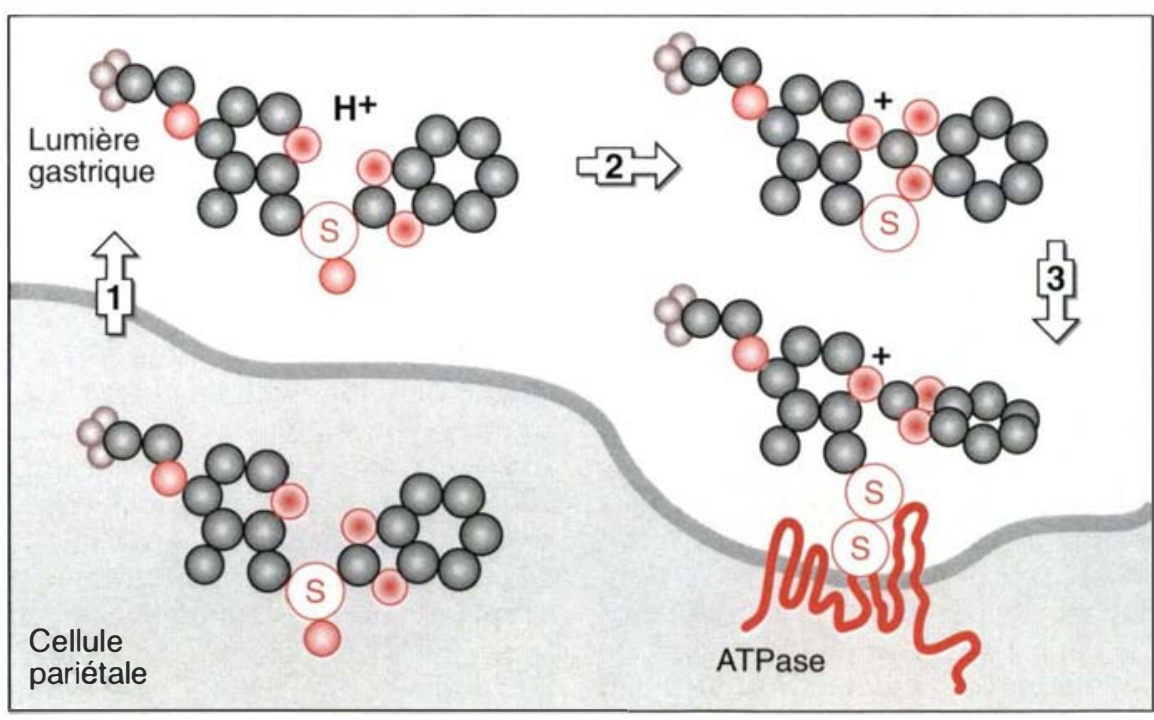

Figure 5. Les trois étapes du mécanisme d'action des IPP lici le lansoprazo(e) : (1) passage du cytosol de la cellule pariétale à la lumière gastrique à travers la membrane du canalicule. Le produit se concentre alors sous forme protonée ; (2) conversion du promédicament en forme sulfénamide active (3) fixation covalente (pont disulfure) à la sous-unité $\alpha$ de l'ATPase $\left(\mathrm{H}^{+}, \mathrm{K}^{+}\right)$. pariétale), les antagonistes du récepteur H2 sont de puissants inhibiteurs de la sécrétion acide gastrique. Cependant, il peut arriver que le blocage du récepteur $\mathrm{H} 2$ soit insuffisant che\% certains malades, soit du fait d'un excès de stimulation vagale, soit du fait de la présence d'une tumeur sécrétant de la gastrine (syndrome de Zollinger-Ellison). Dans ces deux cas, les IPP apportent une nouvelle réponse thérapeutique. En outre, grâce à leur longue durée d'action, la lumière gastrique peut être maintenue plus de seize heures au-dessus de $\mathrm{pH}$ 4 avec une seule prise quotidienne [65-67].

L'inhibition du transport membranaire de $\mathrm{H}^{+}$par les IPP a pour conséquence osmotique une inhibition drastique de la sécrétion d'eau, donc du volume du suc gastrique. Il en résulte une légère inhibition de la sécrétion de pepsinogène, par déf aut d'entraînement liquidien. Par ailleurs, les IPP affectent peu la sécrétion du facteur intrinsèque (ce facteur qui est nécessaire à l'absorption intestinale de la vitamine B12 est produit par la cellule pariétale gastrique, mais par un mécanisme différent du transport membranaire de $\mathrm{H}^{+}$).

La durée du traitement d'attaque de l'ulcère duodénal par les IPP actuellement disponibles est de quatre semaines, avec une posologie journalière de $20 \mathrm{mg}$ (oméprazole) ou de $30 \mathrm{mg}$ (lansoprazole). Ces médicaments se présentent sous la forme de gélules à délitement intestinal (pour éviter qu'ils ne soient détruits par l'acide gastrique) à prendre de préférence le soir pour obtenir une meilleure protection contre la sécrétion acide nocturne, qui n'est pas tamponnée par les aliments. A l'issue de cette cure, près de $95 \%$ des ulcères sont cicatrisés. Un résultat sensiblement équivalent est obtenu avec les inhibiteurs du récepteur $\mathrm{H} 2$, mais au bout de six semaines de traitement seulement, le résultat à quatre semaines n'étant que de $80 \%$ de cicatrisation.

Leur plus grande rapidité d'action, comparée à celle des inhibiteurs du récepteur $\mathrm{H} 2$, rend les IPP particulièrement précieux dans le traitement des ulcères hyperalgiques ou hémorragiques. lls sont $a$ fortiori d'un très grand intérêt dans le traitement des ulcères résistant aux anti-H2. Le coût 


\section{RÉFÉRENCES}

67. Mignon M, Cadiot G, Vallot T, Vatier J, Lewin MJM. Diagnostic strategy in Zollinger-Ellison syndrome. In : Neville AM, ger-Ellison syndrome. In: Neville AM, ne pancreatic tumors. Progress in tumor biology, molecular genetics, diagnosis and therapy. Diagn Oncol 1993 : 37-44.

68. Holt S, Howden CW. Omeprazole. Overview and opinion. Dig Dis Sci 1991 ; 36 : 38593.

69. Mignon M, Vallot T. Acute treatment of duodenal ulcer : experience with lansoprazole. Aliment Pharmacol Ther 1993 ; 7 (suppl 1) : $37-40$.

70. Petite JP, Slama JL, Licht H, Lemerez M, Coste T, Andrieu J. Grimaud JC, Julien H, Dupuis J, Sallerin V. Comparaison du lansoprazole $(30 \mathrm{mg})$ et de l'oméprazole (20 prazole (30 $\mathrm{mg})$ et de l'oméprazole (20
$\mathrm{mg})$ dans le traitement de l'ulcère duodénal. Gastroenterol Clin Biol 1993 ; 17 : 334-40.

71. Galmiche JP, Bruley des Varannes S, Scarpignato $C$. Therapeutic strategy in gastro-oesophageal reflux disease. In : Domschke W, Konturek S], eds. Stomach and its diseases. Berlin : Springer, 1993 : 234-48.

72. Lehy T, Mignon M, Cadiot G, ElowaerBlanc L, Ruszniewski Ph, Lewin MJM, Bonfils $\mathrm{S}$. Gastric endocrine cell behavior in Zollinger-Ellison patients upon long-term potent antisecretory treatment. Gastroenterology $1989 ; 96$ : 1029-40.

73. Lehy T, Cadiot G, Mignon M. Influence of multiple endocrine neoplasia type I on gastric endocrine cells in patients with the gastric endocrine cells in patients with the
Zollinger-Ellison syndrome. Cul $1992 ; 33$ : 1275-8.

74. Cadiot G, Laurent-Puig P, Thuille B, Lehy $T$, Mignon $M$, Olschwang $S$. Is the MEN-1 gene a tumor suppressor gene for fundic argyrophil carcinoid tumors in the Zollinger-Ellison syndrome? Gastroenterology $1993 ; 105$ : $579-82$

75. Modlin IM, Nangia AK. The pathobiology of the human enterochromaffin-like cell. In : Modlin IM ed. Current clinical and scientific perspectives in gastroenterology. New Haven : The Yale Journal of Biology and Medicine, $1992: 361-78$.

76. Lee H, Hakanson R, Karlsson A, Mattsson H, Sundler F. Lansoprazole and omeprazole have similar effects on plasma gastrin levels, enterochromaffin-like cells, gastrin cells and somatostatin cells in the rat stomach. Digestion $1992 ; 51: 125-32$.

77. Hakanson R, Sundler F. Proposed mechanism of induction of gastric carcinoids. The gastrin hypothesis. Eur J Clin Invest du traitement par l'un ou l'autre de ces inhibiteurs est sensiblement le même (autour de 400) F).

En ce qui concerne les ulcères gastriques, plus difficiles à cicatriser, le résultat moyen à quatre semaines est de l'ordre de $60 \%$ de cicatrisation avec les antagonistes de récepteurs H2, de 70 \% avec l'oméprazole (gélules de $20 \mathrm{mg}$ ) et de $75 \%$ avec le lansoprazole (gélules de $30 \mathrm{mg}$ ). Cependant, pour tous ces inhibiteurs, le taux de cicatrisation dépasse $85 \%$ si le traitement est prolongé de quatre semaines supplémentaires [60, 68-70]. I.'intérêt particulier des IPP se manifeste surtout dans le traitement de l'œesophagite par reflux. I a muqueuse œsophagienne est en eff et particulièrement sensible au contact du suc gastrique auquel elle n'est pas préparée, contrairement à la muqueuse gastro-duodénale. Sa capacité de réparation est donc liée de façon encore plus critique que cette dernière à l'efficacité avec laquelle la sécrétion acide est inhibée et à la durée de cette inhibition. Or nous avons vu que les IPP sont, d'une part, extrêmement efficaces et possèdent, d'autre part, une durée d'action très prolongée. Dans le traitement de ces oesophagites, on observe une différence assez nette entre les antagonistes des récepteurs H2, qui donnent, aux posologies classiques, un taux de cicatrisation à quatre semaines de $50 \%$ à $60 \%$ et les IPP dont le taux de cicatrisation dépasse $80 \%$ à quatre semaines et peut atteindre $95 \%$ à huit semaines [60, $71]$.

On doit cependant réaliser que le traitement aigu de la poussée ulcéreuse par un inhibiteur de la sécrétion acide gastrique ne met pas le malade à l'abri d'une rechute : en moyenne, l'ulcère récidive chez deux patients sur trois au bout de un an sans traitement. Dans le cas des œesophagites par reflux, les antisécrétoires ne règlent pas non plus le problème de fond et la maladie reprend dès l'arrêt de la prise de ces médicaments. Pour diminuer la fréquence de ces récidives, on peut envisager de maintenir un traitement d'entretien pendant plusieurs mois, voire plusieurs années. A priori, les IPP, comme les antagonistes des récepteurs H2, se prêtent bien à une administration chronique car ils sont apparemment dénués de toute toxicité. Ce- pendant, les posologies doivent être ajustées pour éviter une inhibition totale et permanente de la sécrétion acide qui risquerait de conduire à l'apparition de deux effets pervers. I.'un est la pullulation dans l'estomac de bactéries de type Colibacille et I'seudomonas susceptibles de produirc des composés potentiellement carcinogènes (N-nitrosoamines) à partir des nitrates et nitrites alimentaires (ces bactéries sont normalement tuées en pH acide). L'autre est l'installation d'une hypergastrinémie élevée due à la perte du frein exercé par l'acide gastrique sur la sécrétion de gastrine des cellules antrales. En effet, la gastrine possède une action mitogène capable de provoquer une hyperprolifération de certaines cellules épithéliales. C'est notamment le cas des cellules ECI. (pour enterochromaffin-like). Ces cellules qui sont localisées dans le fundus gastrique, à proximité des cellules pariétales, jouent un rôle capital dans la stimulation de la sécrétion acide en libérant de l'histamine sous l'action de la gastrine. Une hyperplasie des cellules EC.L est observée dans deux situations pathologiques caractérisées par une hypergastrinémie chronique : le syndrome de Zollinger-Ellison, évoqué plus haut, et l'anémie par défaut de vitamine $\mathrm{B} 12$, dite "pernicieuse" ou "de Biermer", dans laquelle les cellules pariétales sont détruites par des auto-anticorps (et ne produisent donc plus de facteur intrinsèque). Cette hyperplasie disparaît si l'on supprime l'hypergastrinémie, par exemple en ôtant l'antre gastrique chez les sujets atteints de maladie de Biermer. Dans le cadre de ces maladies, on peut également observer des tumeurs "carcinoïdes" gastriques à cellules ECI. [72, 73]. Mais ces tumeurs, de faible malignité, sont tout à fait exceptionnelles et ne semblent apparaître que chez les malades génétiquement prédisposés à développer, de façon générale, des tumeurs de cellules endocrines (syndrome de néoplasie endocrine multiple de type 1 ou "NEM l ") [74].

L'hypothèse de facteurs génétiques facilitant les effets de l'hypergastrinémie est confortée par les observations faites sur Mastomys natalensis. Ce rongeur possède la particularité de produire spontanément des carcinoïdes gastriques vers sa deuxième année 
d'existence, mais si l'on provoque une hypergastrinémie chronique par inhibition de la sécrétion acide, les carcinoïdes apparaissent beaucoup plus tôt (4 à 6 mois) [75]. Néanmoins, la survenue de tumeurs carcinoïdes gastriques a été également rapportée chez le rat à la suite d'un traitement prolongé (deux ans) à très fortes doses d'IPP ou d'anti-H2 et l'hypothèse qu'une hypergastrinémie prolongée puisse à elle seule induire l'apparition de carcinoïdes gastriques ne peut pas être formellement écartée [61, 76, 77]. Ces observations expérimentales ont conduit à porter une attention toute particulière sur les risques potentiels d'une inhibition pharmacologique prolongée de la sécrétion acide chez l'homme. A ce jour, ces risques apparaissent extrêmement faibles puisqu'aucun cas de carcinoïde gastrique imputable aux IPP ou aux anti-H2 n'a été rapporté alors que plusieurs dizaines de millions de patients ont été traités par ces produits.

\section{Conclusions et perspectives}

Les IPP permettent d'inhiber la sécrétion acide gastrique avec une efficacité quasi absolue et une longue durée d'action. Ils sont en outre très spécifiques et apparemment dénués de toute toxicité. Quels progrès peuton encore attendre dans cette classe pharmacologique ? De nouveaux IPP sont en cours de mise au point, mais il semble difficile de dépasser les per- formances pharmacologiques des molécules existant déjà. L'intérêt de cet objectif est d'ailleurs discutable On a vu que l'inhibition trop drastique de la sécrétion acide pouvait conduire à des effets indésirables et il est donc peut-être préférable de permettre à l'estomac de se réacidifier quelques heures par jour. La mise au point d'inhibiteurs réversibles dont nous avons évoqué la possibilité représente, à cet égard, une intéressante alternative, mais on voit mal comment ces inhibiteurs pourraient se démarquer significativement des antagonistes des récepteurs $\mathrm{H} 2$ actuellement disponibles. En conclusion, avec l'avènement des IPP, les médicaments inhibiteurs de la sécrétion acide gastrique paraissent avoir atteint un niveau optimal d'efficacité dans le traitement symptomatique des maladies liées à l'acidité gastrique, comme l'ulcère gastro-duodénal et, particulièrement, l'œesophagite par reflux et le syndrome de Zollinger-Ellison. Ces inhibiteurs ne s'attaquent cependant pas aux causes premières de ces affections qui restent, quant à elles, largement incontrôlées

\section{TIRÉS À PART}

M. J.-M. L.ewin.
* GLOSSAIRE *

ATPase $\left(\mathrm{H}^{+}, \mathrm{K}^{+}\right)$: pompe à protons gastrique

IPP : inhibiteurs de l'ATPase $\left(\mathrm{H}^{+}, \mathrm{K}^{+}\right)$

$\mathrm{H2}$ : récepteur histaminique $\mathrm{H} 2$

MDPQ : 1-(2 méthylphényl)-4 méthylamino-6-méthyl-2,3-dihydropyrrolo [3,2-c] quinoléine

FITC : isothiocyanate de fluorescéine

ANS : aminonaphtol sulfonate de sodium

$C R E$ : cyclic AMP responsive element

$p K$ : constante d'ionisation

$\boldsymbol{E C L}$ : enterochromaffin-like

NEM1: néoplasie endocrine multiple de type 1

\section{Summary}

The inhibitors of the gastric $\left(\mathrm{H}^{+}\right.$, $\mathbf{K}^{+}$) ATPase : mechanism of action and therapeutic efficacy

Over the last twenty years, the treatment of diseases related to gastric acid secretion, such as gastroduodenal ulcer and reflux esophagitis, has greatly improved owing to the development of drugs inhibiting the physiological stimulation of this secretion (histamine $\mathrm{H} 2$ receptor antagonists). The proton pump inhibitors (PPI) constitute a new class of molecules possessing a remarkably prolonged and efficient antisecretory action based on a different mechanism : the blockade of $\mathrm{H}^{+}$transport by the $\left(\mathrm{H}^{+}, \mathrm{K}^{+}\right)$ATPase. 